\title{
openheart CT coronary angiography: a paradigm shift for functional imaging tests
}

\author{
Khaled Alfakih, ${ }^{1,2}$ Jonathan Byrne, ${ }^{2}$ Mark Monaghan ${ }^{2}$
}

To cite: Alfakih K, Byrne J, Monaghan M. CT coronary angiography: a paradigm shift for functional imaging tests. Open Heart 2018;5:e000754. doi:10.1136/ openhrt-2017-000754

Received 30 November 2017 Revised 28 December 2017 Accepted 29 December 2017

Check for updates

${ }^{1}$ Department of Cardiology, University Hospital Lewisham, London, UK

${ }^{2}$ Department of Cardiology, King's College Hospital, London, UK

Correspondence to Dr Khaled Alfakih; khaled. alfakih@nhs.net

\section{ABSTRACT}

The UK National Institute for Health and Care Excellence (NICE) have just updated their guideline on new-onset stable chest pain, recommending that all patients should be investigated with a CT coronary angiography (CTCA). In a separate guideline, NICE recommended CT fractional flow reserve (CT-FFR), to assess coronary stenoses, found on CTCA, stating that this would reduce the need for invasive coronary angiography and hence reduce cost. We discuss the evidence base for CT-FFR and emphasise that we already have established functional imaging tests, with extensive evidence base for efficacy and prognosis and that CT-FFR should be compared with this standard of care and not with the much more expensive and invasive fractional flow reserve undertaken during invasive coronary angiography.

The introduction of CT coronary angiography (CTCA) into clinical practice over a decade ago was described as a paradigm shift in the diagnosis of coronary artery disease (CAD), from functional testing, to investigate for the presence of ischaemia, to anatomical testing to investigate for the presence of atherosclerotic CAD. A large number of studies demonstrated that CTCA has an excellent negative predictive value (NPV), ${ }^{12}$ and hence CTCA was incorporated into international guidelines as an alternative to functional imaging tests in patients with low-intermediate pretest probability of CAD. ${ }^{3}$ The UK National Institute for Health and Care Excellence (NICE) in 2010 recommended cardiac CT for patients with chest pain and low pretest probability of CAD, functional imaging tests for patients with intermediate probability of CAD and invasive coronary angiography (ICA) for patients with high probability of CAD. However, in their 2016 guideline, NICE expanded the role of CTCA to be the first-line investigation for all patients with new-onset typical or atypical chest pain. They stated that functional imaging tests should be reserved for the assessment of patients with chest pain and known CAD and for patients where the CTCA is non-diagnostic or has shown CAD of uncertain significance. ${ }^{4}$ The NICE health economic evaluation and the guideline were influenced by the low cost of CTCA and the excellent NPV, based on studies in populations with relative low prevalence of CAD. ${ }^{2}$

Large clinical trials such as Prospective Multicenter Imaging Study for Evaluation of Chest Pain (PROMISE) $(\mathrm{n}=10000)$, which compared CTCA versus functional imaging tests for the initial assessment of patients with suspected CAD, found no difference in outcome. The average pretest probability risk score (RS) in the trial was $53.3 \%$. However, the actual prevalence of CAD was low with $6.2 \%$ of the CTCA cohort and $3.2 \%$ of the functional imaging test cohort going on to have revascularisation. ${ }^{5}$ The higher rate of revascularisation in the CTCA arm should be considered carefully, as we expand the role of CTCA into populations of higher prevalence of $\mathrm{CAD}$, as it would increase the overall cost to healthcare services, without any evidence of change in outcome, based on PROMISE. This is particularly important as CTCA is known to have a low positive predictive value (PPV). ${ }^{12}$ Hence, a case can be made that patients found to have anatomically significant CAD on CTCA should undergo a test for ischaemia before ICA and revascularisation are considered. CT fractional flow reserve (CT-FFR) has been proposed as this gatekeeper and has now been recommended by NICE, in a separate guideline, for patients with stable chest pain who had a CTCA. The guideline states that CT-FFR may avoid the need for ICA and save the National Health Service a £214 per patient and $£ 9.1$ million by 2022 by reducing the need for ICA. ${ }^{6}$

Several studies have been undertaken comparing CT-FFR with invasive FFR to establish the accuracy of the technique. Determination of Fractional Flow Reserve by Anatomic Computed Tomographic Angiography (DeFACTO) $\quad(\mathrm{n}=252)$ compared CT-FFR with invasive FFR in patients scheduled to have ICA and found the accuracy to be $73 \%$ with sensitivity of $90 \%$, specificity of $54 \%$, PPV of $67 \%$ and NPV of $84 \% .^{7}$ The 
NXT trial $(n=365)$ also compared CT-FFR with invasive FFR in patients scheduled to have ICA. In NXT, only 251 patients, with excellent image quality, were included in the final analysis and the diagnostic accuracy improved to $81 \%$ with sensitivity of $86 \%$, specificity of $79 \%$, PPV of $65 \%$ and NPV of $93 \% .^{8}$

The largest CT-FFR trial to date is the Platform study $(\mathrm{n}=585)$ which compared CTCA plus CT-FFR versus the standard of care. The end point of the study was reduction of ICA that showed no obstructive CAD. The standard of care was unusually skewed towards direct invasive management and in the analysis the patients were divided into two substudies, an invasive study $(\mathrm{n}=380)$ and a non-invasive study $(n=204)$. In the invasive study, CTCA plus CT-FFR reduced the rate of ICA significantly as only patients found to have significant coronary stenosis and positive CT-FFR went on to have ICA, while in the invasive arm of the invasive study all patients, by definition, underwent ICA+invasive FFR. In the non-invasive study, there was no difference in the rate of ICA and revascularisations between functional imaging tests and CTCA plus CT-FFR. The Platform concluded that CTCA plus CT-FFR was associated with a significantly lower rate of ICA showing no obstructive CAD. ${ }^{9}$ Subsequent health economics study concluded that CTCA plus CT-FFR was a more cost-effective approach in the investigation of patients with stable chest pain. ${ }^{10}$ NICE guideline on CT-FFR was based on the Platform health economics data. ${ }^{6}$

In NXT and Platform trials, CT-FFR was performed on all patients with a coronary stenosis on CTCA of $\geq 30 \%$. CT-FFR is likely to perform well in patients with mild stenoses or very severe stenoses. This was demonstrated in a recent systematic review which showed that while the overall diagnostic accuracy of CT-FFR, compared with invasive FFR, was $81.9 \%$, the highest accuracy for CT-FFR was below FFR of 0.60 at $86.4 \%$ and above FFR of 0.80 at $87.3 \%$. The accuracy became very poor in patients with CT-FFR values in the range of $0.70-0.80$ at $46.1 \%$, which is where FFR is most important. ${ }^{11}$ This questions the efficacy of routine use of CT-FFR as a gatekeeper to ICA and revascularisation.

Most recently, Dewey et $a l^{2}$ undertook a clinical trial $(\mathrm{n}=340)$ randomising patients to CTCA versus direct invasive management with ICA and found that CTCA reduced the rate of ICA from $100 \%$ in the invasive arm to $14 \%$ in the CTCA arm. The pretest probabilit y RS of the cohort was $34.6 \%$, which is lower than the PROMISE trial. Demonstrating that CTCA reduces the need for ICA in patients who do not need to be investigated with an ICA is not very meaningful.

We already have well-established functional imaging tests with proven clinical efficacy and extensive prognostic data. ${ }^{13-18}$ The PROMISE trial demonstrated that functional imaging tests lead to fewer revascularisations without a difference in outcome. Functional imaging tests in PROMISE did result, however, in more invasive angiograms that do not lead to revascularisations, compared with CTCA ( $4.3 \%$ vs $3.4 \%)$. At least some of these patients, with false positive functional imaging tests, do in fact have ischaemia, but with microvascular $\mathrm{CAD}$, and they do have an adverse outcome. ${ }^{19}$ This is being investigated in the CIAO arm of the ischaemia trial which will report in $2019 .^{20}$ Functional imaging tests should be the default gatekeeper to ICA and revascularisations, in patients with significant anatomical CAD on CTCA. Studies comparing CTCA plus CT-FFR with ICA plus invasive FFR in these patients do not represent the clinical reality or international guidelines. ${ }^{34}$ CT-FFR needs to be directly compared with functional imaging tests and the health economic evaluations of CT-FFR should be based on such studies. This is particularly important as the UK hospitals have recently had to expand their functional imaging test resources to be able to deliver the NICE 2010 guidelines. The British Society of Cardiovascular Imaging executive summary states that the switch to CTCA for all patients with stable chest pain will require a significant investment in CT scanners and workforce. ${ }^{21}$ The tariff of CT-FFR is an additional $£ 700$ per patient, on top of the cost of the CTCA. This is significantly higher than the tariff for a functional imaging test such as stress echo at $£ 270$, which has the added advantage of an extensive evidence base. $^{13-18}$

Contributors All three authors contributed to the idea and content. KA wrote the manuscript. MM and JB critiqued the manuscript.

Competing interests None declared.

Patient consent Not required.

Provenance and peer review Not commissioned; externally peer reviewed.

Data sharing statement There are no additional data available for this article.

Open Access This is an Open Access article distributed in accordance with the Creative Commons Attribution Non Commercial (CC BY-NC 4.0) license, which permits others to distribute, remix, adapt, build upon this work non-commercially, and license their derivative works on different terms, provided the original work is properly cited and the use is non-commercial. See: http://creativecommons.org/ licenses/by-nc/4.0/

(c) Article author(s) (or their employer(s) unless otherwise stated in the text of the article) 2018. All rights reserved. No commercial use is permitted unless otherwise expressly granted.

\section{REFERENCES}

1. Mowatt $G$, Cummins $E$, Waugh $N$, et al. Systematic review of the clinical effectiveness and cost-effectiveness of 64-slice or higher computed tomography angiography as an alternative to invasive coronary angiography in the investigation of coronary artery disease. Health Technol Assess 2008;12:iii-iv, ix-143.

2. Budoff MJ, Dowe D, Jollis JG, et al. Diagnostic performance of 64-multidetector row coronary computed tomographic angiography for evaluation of coronary artery stenosis in individuals without known coronary artery disease: results from the prospective multicenter ACCURACY (Assessment by Coronary Computed Tomographic Angiography of Individuals Undergoing Invasive Coronary Angiography) trial. J Am Coll Cardiol 2008;52:1724-32.

3. Montalescot G, Sechtem U, Achenbach S, et al. 2013 ESC guidelines on the management of stable coronary artery disease: the Task Force on the management of stable coronary artery disease of the European Society of Cardiology. Eur Heart $J$ 2013;34:2949-3003.

4. NICE. Chest pain of recent onset: assessment and diagnosis. London, UK: NICE, 2017.

5. Douglas PS, Hoffmann U, Patel MR, et al. Outcomes of anatomical versus functional testing for coronary artery disease. N Engl J Med 2015;372:1291-300.

6. NICE. HeartFlow FFRCT for estimating fractional flow reserve from coronary CT angiography. 2017. https://www.nice.org.uk/guidance/ mtg32/chapter/1-Recommendations 
7. Min JK, Leipsic J, Pencina MJ, et al. Diagnostic accuracy of fractional flow reserve from anatomic CT angiography. JAMA 2012;308:1237-45.

8. Nørgaard BL, Leipsic J, Gaur S, et al. Diagnostic performance of noninvasive fractional flow reserve derived from coronary computed tomography angiography in suspected coronary artery disease: the NXT trial (Analysis of Coronary Blood Flow Using CT Angiography: Next Steps). J Am Coll Cardiol 2014;63:1145-55.

9. Douglas PS, Pontone G, Hlatky MA, et al. Clinical outcomes of fractional flow reserve by computed tomographic angiographyguided diagnostic strategies vs. usual care in patients with suspected coronary artery disease: the prospective longitudinal trial of FFR(CT): outcome and resource impacts study. Eur Heart $J$ 2015;36:3359-67.

10. Hlatky MA, De Bruyne B, Pontone G, et al. Quality-of-life and economic outcomes of assessing fractional flow reserve with computed tomography angiography: PLATFORM. J Am Coll Cardiol 2015;66:2315-23.

11. Cook CM, Petraco R, Shun-Shin MJ, et al. Diagnostic accuracy of computed tomography-derived fractional flow reserve : a systematic review. JAMA Cardiol 2017;2:803-10.

12. Dewey M, Rief M, Martus P, et al. Evaluation of computed tomography in patients with atypical angina or chest pain clinically referred for invasive coronary angiography: randomised controlled trial. BMJ 2016;355:i5441
13. Marcovitz PA, Armstrong WF. Accuracy of dobutamine stress echocardiography in detecting coronary artery disease. Am J Cardiol 1992;69:1269-73.

14. Ryan T, Vasey CG, Presti CF, et al. Exercise echocardiography: detection of coronary artery disease in patients with normal left ventricular wall motion at rest. J Am Coll Cardiol 1988;11:993-9.

15. Segar DS, Brown SE, Sawada SG, et al. Dobutamine stress echocardiography: correlation with coronary lesion severity as determined by quantitative angiography. J Am Coll Cardiol 1992;19:1197-202

16. Sicari R, Pasanisi E, Venneri L, et al. Stress echo results predict mortality: a large-scale multicenter prospective international study. $J$ Am Coll Cardiol 2003;41:589-95.

17. Marwick TH, Case C, Sawada S, et al. Prediction of mortality using dobutamine echocardiography. J Am Coll Cardiol 2001;37:754-60.

18. Poldermans D, Fioretti PM, Boersma E, et al. Long-term prognostic value of dobutamine-atropine stress echocardiography in 1737 patients with known or suspected coronary artery disease: A singlecenter experience. Circulation 1999;99:757-62.

19. Papachristidis A, Roper D, Demarco D, et al. The utility and outcomes of Stress Echo in a contemporary high volume tertiary centre. Echo Res Pract 2016;4:105-13.

20. ISCHEMIA. For physicians. 2018. https://www.ischemiatrial.org/ physicians

21. BSCI/BSCCT. The British Society of Cardiovascular Imaging and British Society of Cardiovascular CT. 2016. http://www.bsci.org. uk/standards-guidelines/nice-cg95-update-2016?id=174 